\title{
Energy dependence of moments of the net-charge multiplicity distributions in Au+Au collisions at RHIC
}

\author{
Dipak K. Mishra* (for the PHENIX Collaboration) \\ Nuclear Physics Division \\ Bhabha Atomic Research Center \\ Mumbai, India - 400085 \\ E-mail: dkmishra@rcf.rhic.bnl.gov
}

\begin{abstract}
The Beam Energy Scan program at the Relativistic Heavy Ion Collider (RHIC) is aimed to probe the QCD phase diagram in the vicinity of a possible critical point. Higher moments of multiplicity distributions of conserved quantities are predicted to be sensitive to the fluctuations due to critical point. Lattice QCD predictions and statistical thermal models suggest that the susceptibility of the medium formed in heavy-ion collisions can be related to various moments (Mean $(\mu)=\langle x\rangle$, Variance $\left(\sigma^{2}\right)=\left\langle(x-\mu)^{2}\right\rangle$, Skewness $(S)=\left\langle(x-\mu)^{3}\right\rangle / \sigma^{3}$ and Kurtosis $(\kappa)=$ $\left\langle(x-\mu)^{4}\right\rangle / \sigma^{4}-3$, of the conserved quantities like net-baryon number $(\Delta B)$, net-electric charge $(\Delta Q)$ and net-strangeness $(\Delta S)$. Non monotonic deviation in the higher moments from the baseline predictions would indicate the existence of the QCD critical point. We present various moments and their products for the net-charge multiplicity distributions in $\mathrm{Au}+\mathrm{Au}$ collisions at different energies ranging from $\sqrt{s_{N N}}=7.7$ to $200 \mathrm{GeV}$, measured by PHENIX experiment at RHIC. The experimental results are compared with different heavy-ion collision models, which do not include the QCD phase transition and critical point.
\end{abstract}

7th International Conference on Physics and Astrophysics of Quark Gluon Plasma

1-5 February, 2015

Kolkata, India

${ }^{*}$ Speaker. 


\section{Introduction}

Recent calculations predict the existence of the critical end point (CEP) in the QCD phase diagram $[1,2]$. The point at which the first order phase transition ends in the $T-\mu_{B}$ plane is known as CEP. The beam energy scan program at RHIC provided large amount of data at different collision energies, which gives us an unique opportunity to study the location and existence of CEP in the QCD phase diagram. In the thermodynamic limit, the correlation length $\xi$ diverges at CEP [3]. The correlation length is related to various moments of conserved quantities such as: net-baryon, net-charge or net-strangeness number. The non-monotonic behavior in the fluctuation of these conserved quantities as a function of collision energies $\left(\sqrt{s_{N N}}\right)$ is one of the signatures to locate CEP [4]. Various moments of the conserved quantities are related to the different orders of correlation length as: variance $\left(\sigma^{2}\right)=\left\langle(\delta N)^{2}\right\rangle \sim \xi^{2}$, skewness $(S)=\left\langle(\delta N)^{3}\right\rangle / \sigma^{2} \sim \xi^{4.5}$ and kurtosis $(\kappa)=\left\langle(\delta N)^{4}\right\rangle / \sigma^{4}-3 \sim \xi^{7}$, where $\delta N=N-M$, with $N$ is multiplicity and $M$ being the mean of the distribution. Since higher moments have stronger dependence on $\xi$, hence can have better sensitivity to CEP. Lattice calculations and other thermal models such as HRG show that moments of the conserved quantities are related to the susceptibilities $(\chi)$ associated with the conserved number as $\sigma^{2} / M \sim \chi^{2} / \chi^{1}, S \sigma \sim \chi^{3} / \chi^{2}$ and $\kappa \sigma^{2} \sim \chi^{4} / \chi^{2}[5,6]$. One advantage of measuring $\sigma^{2} / M, S \sigma$ and $\kappa \sigma^{2}$ is that the volume dependence of individual moment cancels out in the ratios, hence experimentally measured quantities can be compared with the theoretical calculations. In recent studies, it has been shown the $\sigma^{2} / M$ and $S \sigma$ can be used to extract the freeze-out parameters $\left(T_{f}\right.$ and $\left.\mu_{B}\right)$ in heavy ion collisions [5].

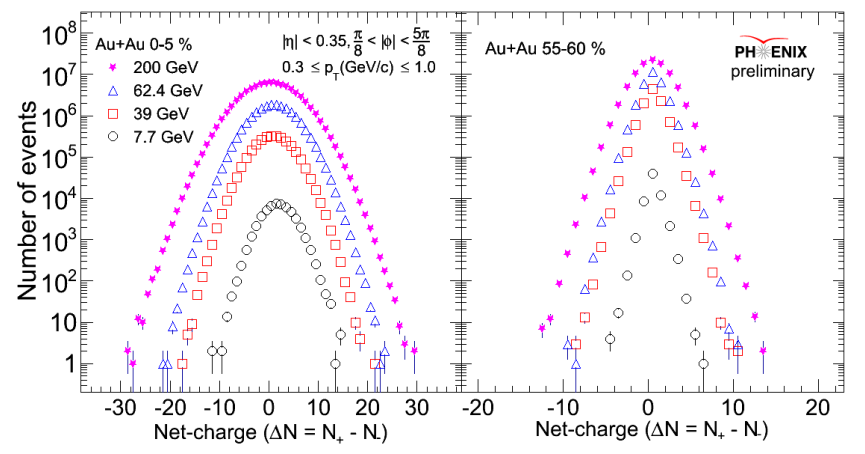

Figure 1: Uncorrected net-charge $(\Delta N)$ distributions measured on event-by-event basis for $\mathrm{Au}+\mathrm{Au}$ collisions at different energies for two different centralities (0-5\% and 55-60\%) within $|\eta|<0.35$.

\section{Analysis}

In this report, we present the higher moments of net-charge multiplicity distributions in $\mathrm{Au}+\mathrm{Au}$ collisions at $\sqrt{s_{N N}}=7.7,39,62.4$ and $200 \mathrm{GeV}$ measured by PHENIX experiment at RHIC. To measure the net-charge $\left(\Delta N=N^{+}-N^{-}\right)$distributions, the number of positively charged $\left(N^{+}\right)$and negatively charged $\left(N^{-}\right)$particles are measured on an event-by-event basis within $\pm 30 \mathrm{~cm}$ along the beam direction of the collision vertex. The charged particle tracks are reconstructed using informations from the drift chamber and pad chambers [7]. Tracks having a transverse momentum 
$\left(p_{T}\right)$ between 0.3 and $1.0 \mathrm{GeV} / c$ and pseudo-rapidity $(|\eta| \leq 0.35)$ are selected for the analysis. The standard PHENIX track quality cuts are applied for the analysis. In order to avoid the autocorrelation effect in analyzed tracks, the collision centrality selection has been done by using tracks from a different $|\eta|$ range. The statistical uncertainties have been calculated using Delta theorem method [8].
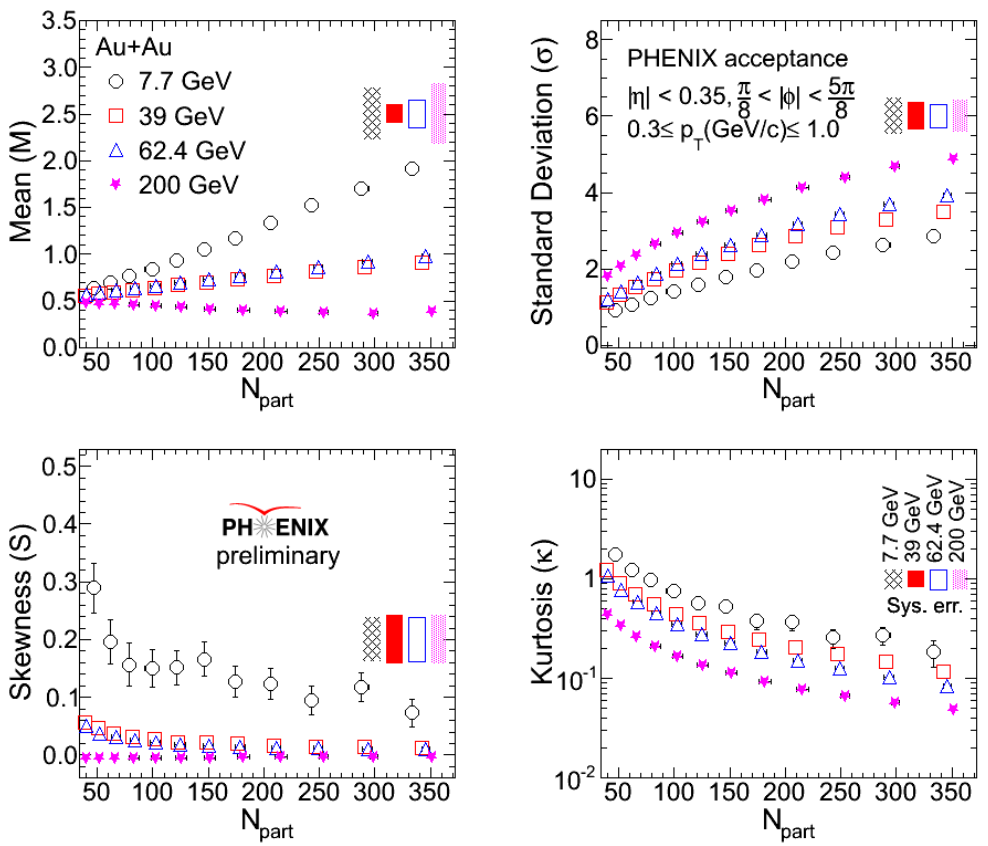

Figure 2: Efficiency uncorrected moments $M, \sigma, S$ and $\kappa$ obtained from net-charge distributions as a function of centrality $\left(N_{\text {part }}\right)$ from $\mathrm{Au}+\mathrm{Au}$ collisions at different collision energies from the PHENIX experimental data. The statistical uncertainties are shown along with the data points. The scaled systematic uncertainties are shown in shaded.

\section{Results}

Figure 1 shows the uncorrected $\Delta N_{c h}$ distributions in $\mathrm{Au}+\mathrm{Au}$ collisions for central (0-5)\% and peripheral (55-60)\% collisions at different collision energies. The $\Delta N_{c h}$ distributions are symmetric around mean value. Various moments are calculated from the above distributions. Figure 2 shows the efficiency uncorrected $M, \sigma, S$ and $\kappa$ as a function of collision centralities $\left(N_{\text {part }}\right)$ extracted from the net-charge distributions at different $\sqrt{S_{N N}}$. The moments are corrected for finite centrality bin width effect. The mean and standard deviation increase with increasing $N_{\text {part }}$, while $S$ and $\kappa$ decrease with increasing $N_{\text {part }}$ for all collision energies. In order to remove the volume dependence of moments so that the experimental values can be compared with the model calculations, the product $\kappa \sigma^{2}$ and $S \sigma$ are used. Figure 3 shows the centrality dependence of $S \sigma$ and $\kappa \sigma^{2}$ for different collision energies. It is observed from Fig. 3 that, $S \sigma$ and $\kappa \sigma^{2}$ values are weakly dependent on $N_{\text {part }}$. The values of $S \sigma$ decrease from lower to higher collision energies, where as $\kappa \sigma^{2}$ is constant 


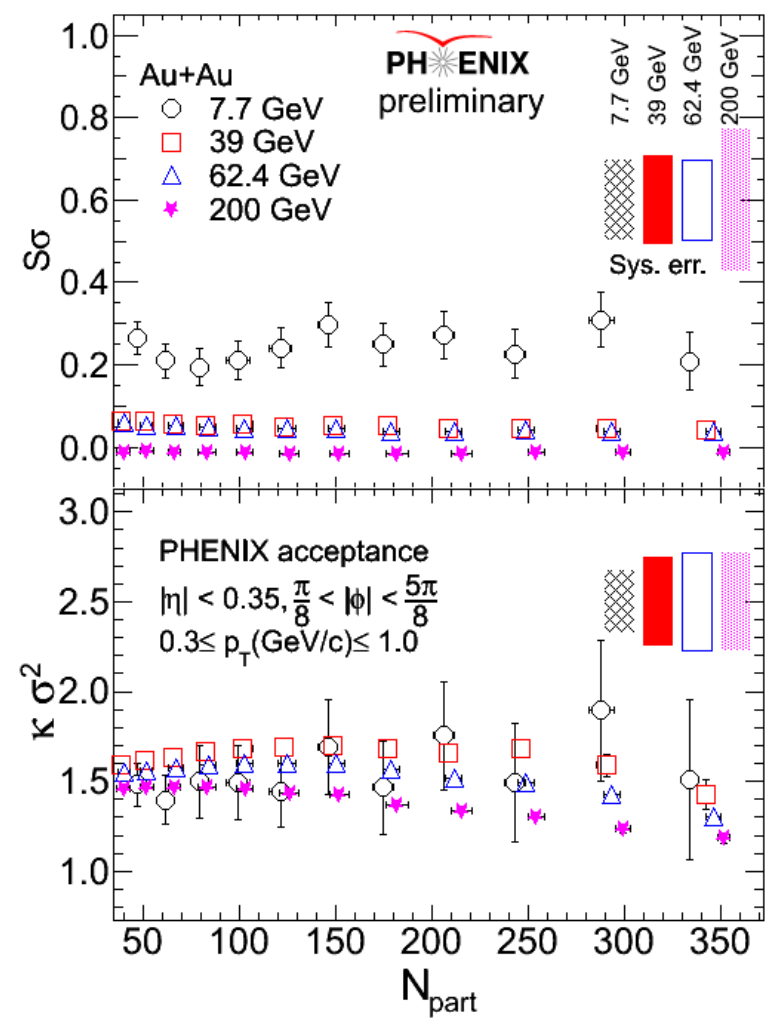

Figure 3: Centrality dependence of efficiency uncorrected $\sigma^{2} / M, S \sigma$ and $\kappa \sigma^{2}$ of net-charge distributions for $\mathrm{Au}+\mathrm{Au}$ collisions at different collision energies. Statistical errors are shown along with the data points while scaled systematic uncertainties are shown in shaded region.

for all $\sqrt{s_{N N}}$ within systematic uncertainties. Figure 4 shows the energy dependence of $\sigma^{2} / M$, $S \sigma$ and $\kappa \sigma^{2}$ of the net-charge distributions for most central (0-5)\% collisions. The experimental results are compared with the non-CEP based models like HRG, UrQMD and HIJING simulation results within the PHENIX acceptance. The $\sigma^{2} / M$ values increase with increasing beam energies. The $\sigma^{2} / M$ values from HIJING model over estimate the experimental data at all energies. The $S \sigma$ values increase with decreasing $\sqrt{S_{N N}}$ where as $\kappa \sigma^{2}$ values remain constant over all collision energies within uncertainties. The $S \sigma$ and $\kappa \sigma^{2}$ values obtained from UrQMD and HIJING models agree with the experimental data except for $\sqrt{s_{N N}}=200 \mathrm{GeV}$, which might be due to the more resonance production at higher energies. No excess is observed in the analyzed data at these four collision energies above the simulation results.

\section{Summary and Outlook}

Fluctuation of net-charge multiplicity distributions have been measured for $\mathrm{Au}+\mathrm{Au}$ collisions at $\sqrt{s_{N N}}=7.7,39,62.4$ and $200 \mathrm{GeV}$ by the PHENIX experiment. Various moments $(M, \sigma, S, \kappa)$ are extracted from $\Delta N_{c h}$ distributions and are studied as a function of $N_{\text {part }}$ for all collision energies. The $\sigma^{2} / M$ values increase with $\sqrt{s_{N N}}$, where as $S \sigma$ values increase with decreasing collision 


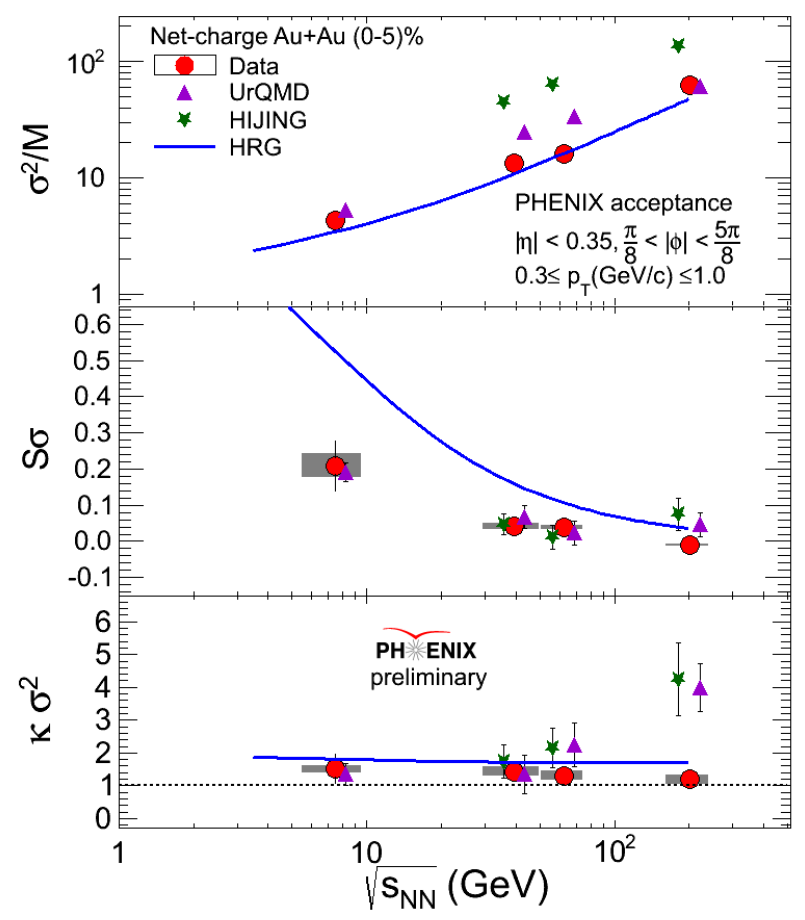

Figure 4: Energy dependence of efficiency uncorrected $\sigma^{2} / M, S \sigma$ and $\kappa \sigma^{2}$ of net-charge distributions for central (0-5)\% Au+Au collisions from the PHENIX experimental data. The statistical and systematic (shaded) uncertainties are also shown with the data points. The experimental values are compared with the HRG, UrQMD and HIJING models.

energies. The $\kappa \sigma^{2}$ values remain constant over all collision energies. The experimental values are compared with the non-CEP based models. In the presented results from four collision energies we don't observe any significant deviation from the model calculations. It will be interesting to see the results at lower $\sqrt{s_{N N}}$ at 19.6 and $27 \mathrm{GeV}$. However, by comparing these results with the lattice calculations will provide very useful information to extract the freeze-out parameters.

\section{References}

[1] Z. Fodor and S. D. Katz, J. High Energy Phys. 04050 (2004)

[2] M. A. Stephanov, K. Rajagopal and E. V. Shuryak, Phys. Rev. D 60, 114028 (1999) [hep-ph/9903292].

[3] M. A. Stephanov, K. Rajagopal and E. V. Shuryak, Phys. Rev. Lett. 81, 4816 (1998) [hep-ph/9806219].

[4] V. Koch, A. Majumder and J. Randrup, Phys. Rev. Lett. 95, 182301 (2005) [nucl-th/0505052].

[5] A. Bazavov, H. T. Ding, P. Hegde, O. Kaczmarek, F. Karsch, E. Laermann, S. Mukherjee and P. Petreczky et al., Phys. Rev. Lett. 109, 192302 (2012) [arXiv:1208.1220 [hep-lat]].

[6] F. Karsch and K. Redlich, Phys. Lett. B 695, 136 (2011) [arXiv:1007.2581 [hep-ph]].

[7] K. Adcox et al. [PHENIX Collaboration], Nucl. Instrum. Meth. A 499, 469 (2003).

[8] X. Luo, J. Phys. G 39, 025008 (2012) [arXiv:1109.0593 [physics.data-an]]. 